\title{
1 Modeling and simulation of RF photoinjectors for coherent light sources
}

2 Y. Chen ${ }^{1}$, M. Kraslinikov and F. Stephan

Deutsches Elektronen Synchrotron DESY, Platanenallee 6, 15738 Zeuthen, Germany

E. Gjonaj and T. Weiland

TEMF, Technische Universitaet Darmstadt, Schlossgartenstr. 8, 64289 Darmstadt, Germany

M. Dohlus

Deutsches Elektronen Synchrotron DESY, Notkestrae 85, 22607 Hamburg, Germany

\begin{abstract}
We propose a three-dimensional fully electromagnetic numerical approach for the simulation of RF photoinjectors for coherent light sources. The basic idea consists in incorporating a selfconsistent photoemission model within a particle tracking code. The generation of electron beams in the injector is determined by the quantum efficiency $(\mathrm{QE})$ of the cathode, the intensity profile of the driving laser as well as by the accelerating field and magnetic focusing conditions in the gun. The total charge emitted during an emission cycle can be limited by the space charge field at the cathode. Furthermore, the time and space dependent electromagnetic field at the cathode may induce a transient modulation of the QE due to surface barrier reduction of the emitting layer. In our modeling approach, all these effects are taken into account. The beam particles are generated dynamically according to the local QE of the cathode and the time dependent laser intensity profile. For the beam dynamics, a tracking code based on the LienardWiechert retarded field formalism is employed. This code provides the single particle trajectories as well as the transient space charge field distribution at the cathode. As an application, the PITZ injector is considered. Extensive electron bunch emission simulations are carried out for different operation conditions of the injector, in the source limited as well as in the space charge limited emission regime. In both cases, fairly good agreement between measurements and simulations is obtained.
\end{abstract}

Keywords: RF photoinjector, emission, quantum efficiency, space charge, Lienard-Wiechert.

\section{Introduction}

RF photoinjectors can provide highly charged electron beams with very low transverse emittance [1-8]. This is a crucial property, in particular, for coherent light sources since the radiated light intensity tends to grow near-quadratically with decreasing beam emittance [9]. Therefore, the optimization of injector operation in terms of increasing beam current while at the same time reducing its transverse emittance is an important task in linear accelerator design. This task includes, in the first place, the characterization of the electron beam with respect to a large number of machine parameters such as photocathode material, spot size and intensity of the

\footnotetext{
${ }^{1}$ Corresponding author, ye.lining.chen@desy.de, TEL +49-40-8998-7254, FAX +49 33762 7-7330
} 
cathode-illuminating laser pulse, longitudinal beam profile, accelerating field strength, solenoid current and many others.

Photoinjector characterization studies are typically done by numerical simulations. Several particle tracking tools are available and are commonly used for this purpose (cf. $[5,10-$ 19]). However, so far no suitable numerical model can incorporate the photoemission process with sufficient accuracy to resolve the complex beam dynamics in the close vicinity of the cathode. In other words, it is not possible to predict from first principles the beam current produced by the photocathode for different injector operation conditions. The knowledge of this current is, however, the first prerequisite for all beam tracking and space charge field simulations in the injector and further down in the accelerator chain. A common assumption used in conventional simulations is that the emission current profile is identical with the intensity profile of the laser pulse applied at the cathode. However, the QE of the photocathode, and thus the emission current depends also on the local space charge and RF fields at the cathode (see details in Section 4). This is due to the modulation of the effective work function of the emitting layer by the electric fields applied on the cathode (Schottky-like effect). The space charge field at the cathode is determined itself by the emission current and, furthermore, by the dynamics of the emitted particles in the vicinity of the photocathode. In photoinjectors, the particle dynamics immediately after their emission is characterized by an extremely fast transition from the nonrelativistic to the highly relativistic regime. This makes space charge and beam tracking simulations in this region particularly difficult. The problem is, thus, multifaceted and it requires a numerical model which is able to cope with all of these effects. In the following, we will introduce a simulation approach dealing with these beam dynamics and particle emission issues for RF photoinjectors operated in the source limited as well as in the space charge limited emission regime. We will refer primarily to the PITZ photoinjector of DESY [1-3]. For this injector, a large database of measurements exists which can be used to assess the validity of our simulations.

The paper is organized as follows. In Section 2, a motivation for this work is given by describing the discrepancies arising between conventional beam dynamic simulations and measurements for the PITZ photoinjector at DESY in Zeuthen [20-21]. In Section 3, the particle tracking codes used in the photoemission studies are described. These include a LienardWiechert (LW) based tracking code $[13,22]$ and a fully electromagnetic Particle-In-Cell (PIC) code [14]. The photoemission models used in the simulations are introduced in Section 4. In Section 5, simulation results are presented. These results are compared with corresponding measurements for different operation conditions of the PITZ photoinjector.

\section{The PITZ photoinjector}

The main motivation for this investigation is a number of discrepancies between simulations and measurements, which were observed for the photoinjector of the European XFEL at the Photo Injector Test Facility at DESY in Zeuthen (PITZ) [20-24]. A sketch of the RF photogun employed at PITZ is shown in Fig. 1. Its main components are a 1.6-cell copper cavity operated at $1.3 \mathrm{GHz}$ and a magnetic focusing system. The electron beam is generated at the backplane of the gun cavity by illuminating a cesium-telluride photocathode with short laser pulses. A peak accelerating field of up to $60 \mathrm{MV} / \mathrm{m}$ is applied in the cavity. As a result, electron bunches are accelerated immediately after their emission up to nearly the speed of light within a distance of a few millimeters from the cathode. We will not go into further details on the PITZ gun and its operation parameters. For a thorough description, the interested reader is referred to [1-3]. 
For the characterization of injector current, two typical measurements are performed. One is the measurement of the emitted bunch charge $\left(Q_{t o t}\right)$ as a function of the cathode laser launch phase $\left(\phi_{l}\right)$ for a given laser pulse energy $\left(W_{l}\right)$. The other one is the bunch charge measurement for different laser pulse energies while keeping the laser launch phase constant. In the measurements, Faraday cups (FCs) and integrating current transformers (ICTs) are used. These are located at about $0.78 \mathrm{~m}$ and $0.935 \mathrm{~m}$ downstream of the cathode, respectively. The ICTs can measure the total charge in the electron bunch with a precision of about $30 \mathrm{pC}$ whereas the FCs have a charge resolution of up to $2 \mathrm{pC}$.

A typical phase scan for the PITZ gun is shown in Fig. 2 (see also [20-21]). The gun phase is measured with respect to the maximum mean momentum gain (MMMG) phase. Thus, each gun phase corresponds to a different accelerating field strength applied on the cathode during the bunch emission phase. At the nominal phase ( 0 degree w.r.t. MMMG) and for a RMS laser spot size (XYrms) of $0.3 \mathrm{~mm}$ the charge of the emitted bunch amounts to $1 \mathrm{nC}$. The phase scan measurements are performed for two different laser pulse intensities. For a laser transmission (LT) of $100 \%$, the emitted charge depends mainly on the accelerating electric field gradient at the cathode plane, where the laser intensity is no longer a limiting factor. Thus, the bunch charge measured in this case (top black curve) represents the space charge saturation limit of the gun at the corresponding accelerating field strength. In comparison, a laser transmission of $62 \%$ is defined such in the experiments that a nominal bunch charge of $1 \mathrm{nC}$ can be extracted at 6 degrees w.r.t. MMMG phase of the gun. For the lower laser intensity with LT $=62 \%$ (green curve) and for gun phases between 0 and 50 degrees the bunch charge is nearly constant with respect to the gun phase. In this region, the injector is apparently operated in the source limited regime. In this case, the emitted charge depends only on the laser intensity and on the QE of the cathode, but not on the accelerating field strength at the cathode.

Also in Fig. 2, conventional beam dynamics simulation results obtained from ASTRA [15] (pink, yellow and blue curves) are compared with the measurement data (black and green curves). Since the photoemission process is not explicitly modeled, the charge produced by the cathode is not known a priory and must be provided as an external parameter to the simulation. Thus, the source limited case $(\mathrm{LT}=62 \%$ ) cannot be reproduced exactly in the simulation. Using a fixed input charge $Q_{0}=1.1 \mathrm{nC}$ in the simulation, results in the yellow curve shown in the figure. If the input charge provided at the cathode is increased to $Q_{0}=1.774 \mathrm{nC}$, some of the emitted particles will be pushed back by the space charge field of the bunch, and will then be absorbed in the cavity wall. The remaining particles are injected into the cavity. Their charge represents the space charge saturation limit of the gun corresponding to the case LT $=100 \%$. This limit is depicted by the pink curve in Fig. 2.

We observe that the simulated space charge limit is systematically lower than the measurement. At the nominal gun phase, the bunch charge obtained in the simulation for LT = $100 \%$ and a RMS laser spot size of $0.3 \mathrm{~mm}$ is about $0.85 \mathrm{nC}$. This charge is about $15 \%$ lower than the measured value. Moving towards higher gun phases, the difference between the simulated and measured space charge limit becomes even larger. In some previous studies [20$21,24]$, the RMS spot size was artificially increased to $0.4 \mathrm{~mm}$ in order to obtain the correct value of $1 \mathrm{nC}$ for the total bunch charge at the nominal gun phase. The blue curve in Fig. 2 depicts the charge extracted from the gun for XYrms $=0.4 \mathrm{~mm}$ assuming that the total charge provided by the cathode is exactly $1.1 \mathrm{nC}$. However, this assumption barely shifts the discrepancy to other gun phases. 
Another comparison between measurement and simulation is shown in Fig. 3 [23]. In the figure, the extracted bunch charge is shown for different laser pulse intensities while keeping the RF gun phase fixed to the MMMG phase and the laser spot size to $0.3 \mathrm{~mm}$. The source limited region at lower laser intensities and the space charge limited region at higher intensities, respectively, are clearly recognized. Again, the charge saturation level predicted by the simulation (blue curve) is lower than in the measurement. Moreover, the measured charge continues to increase slightly even for higher laser pulse intensities.

\section{Tracking codes}

A commonly used approach in beam dynamics simulations is based on a quasi-static approximation for the electromagnetic fields. The space charge field is computed in the rest frame of the bunch and is, then, Lorentz-transformed to the laboratory frame, where the particle positions and momenta are updated in every time step of the simulation. This approach is used in tracking codes such as Astra [15], Parmela [16], Impact-T [17], GPT [18] and others. In the following, we will refer to this approach as the Uniform Motion in the Average Frame (UMAF) approximation. This is because, for the space charge field calculation, all the particles in the bunch are assumed to have the same kinetic energy.

A more accurate tracking approach is based on the Lienard-Wiechert (LW) solution for the electromagnetic field of a charged particle in arbitrary relativistic motion. In this approach, the full particle trajectory is stored. This trajectory is, then, used in the computation of LienardWiechert fields. The numerical accuracy is only dependent on the number of simulation particles and on the tracking time step. This method is implemented, e.g., in the code TREDI [19] and in [25-26]. For the purpose of this investigation, we have implemented a new parallelized LW tracking code (cf. [13] for a detailed description). This code is, furthermore, extended to include the emission models described in Section 4.

A third simulation tool used in the following is CST Particle Studio ${ }^{\circledast}$ (CST-PS) [14]. CST-PS is an electromagnetic PIC (EM-PIC) code based on the solution of the full Maxwell equations on the grid. In Fig. 4, using CST-PS and the LW code, the total emitted bunch charge is calculated and compared to the simulation results with the UMAF approach. As shown, the bunch charge calculated by the UMAF approach (dashed blue curve) is limited at below $1 \mathrm{nC}$ for a RMS cathode laser spot size of $0.3 \mathrm{~mm}$. However, no such space charge emission limitation is observed in the full electromagnetic CST-PS and LW simulations (dotted curves and orange curve) for extracting the same bunch, as the full nominal bunch charge of $1 \mathrm{nC}$ can be extracted from the emission process. Previous experiments have shown, furthermore, that it is possible to create a charge packet of $1 \mathrm{nC}$ from the injector for a cathode laser spot size of $0.3 \mathrm{~mm}$ using same gun operation conditions [21-23]. This suggests that the UMAF simulation may underestimate the total bunch charge. Note in addition that, CST-PS as well as the UMAF based codes do not provide a dedicated emission model for the photocathode. Thus, we only use the LW approach for comparing simulation results with the measurement data in Sections 4 and 5.

\section{Source limited emission}

The modeling of the source limited emission regime is based on the classical photoemission theory for the QE of the cathode [27-37]. For metals the Fowler-Dubridge model reads [31-32]:

$$
Q E_{F}=\eta\left(h v-\Phi_{\omega}\right)^{2},
$$


where $h v$ is the photon energy, $\Phi_{\omega}$ is the cathode work function and $\eta$ is a parameter characterizing the cathode. For semiconductors, the Spicer model [27-30] is given by,

$$
Q E_{S}=\frac{\left(h v-\Phi_{\omega}\right)^{1.5}}{\left(h v-\Phi_{\omega}\right)^{1.5}+\gamma} .
$$

Here, $\Phi_{\omega}$ is defined as the sum of the energy band gap $E_{g}$ and the electron affinity $E_{a}$, where the latter is measured from the conduction band minimum [33-34]. The term $\gamma$ is the fitting parameter to the measurement data.

A more recent model for the QE of semiconductor cathodes is the Jensen model [34-37]:

$$
Q E_{J}=\frac{\left(1-R_{\omega}\right) \sqrt{1+\frac{h v-\Phi_{\omega}}{E_{a}}}}{2\left(p_{0}+1\right)\left(1+\frac{E_{a}}{h v-\Phi_{\omega}}\right)^{2}} .
$$

Here, $R_{\omega}$ represents the reflection factor and $p_{0}$ is a free parameter determined by fitting to the experimental data. The term $\Phi_{\omega}$ is denoted as the sum of the energy band gap $E_{g}$ and the electron affinity $E_{a}$.

Note that each of the models Eq. 1 - Eq. 3 involves at least one free parameter describing the combined effects of surface reflectivity, collision frequency and escape probability of the photoelectrons in the emitting layer (see [27-32, 35-37]) for a detailed description of these models). These parameters depend on the material and surface properties of the cathode and, therefore, can only be determined experimentally.

The cathode QE is affected by two more specific mechanisms. The first one concerns the modification of the work function due to the strong electromagnetic fields applied on the cathode [38-39]. The total electric field at the cathode is given by the RF field in the gun, $E_{r f}$, and by the space charge field of the bunch, $E_{s c}$. The applied RF field is reduced by the space charge field lowering the total electric field at the cathode. Taking into account these two contributions, the effective work function of the cathode will be lowered by

$$
\Delta \Phi_{f}\left(r_{\perp}, t\right)=\sqrt{\frac{q^{3}}{4 \pi \varepsilon_{0}}\left[E_{r f}\left(r_{\perp}, t\right)+E_{s c}\left(r_{\perp}, t\right)\right]}
$$

where $r_{\perp}$ is the radial coordinate on the cathode plane. Obviously, the QE of the cathode becomes now time and space dependent. Thus, the emitted bunch charge distribution does not necessarily follow the intensity of the laser pulse applied on the cathode.

A second nonlinear effect is due to the accumulation of trapped electrons within the band-bending region for semiconductor cathodes at higher laser intensities. This charge accumulation at the cathode surface reduces the level of band-bending, resulting in an increase of electron affinity [40]. Consequently, the effective work function is increased and the QE of the cathode is reduced. This effect is sometimes referred as the surface charge limit [41]. We model this effect by introducing a second modification of the effective work function as

$$
\Delta \Phi_{p}\left(r_{\perp}, t\right)=k\left[I\left(r_{\perp}, t\right)\right]^{n}
$$

where $I$ is the transient laser intensity and $k$ and $n$ are model parameters. In [40], a non-linearity coefficient $n=0.5$ is suggested.

Putting these contributions together, the effective work function of the cathode is modified as

$$
\Phi_{\omega}^{\prime}\left(r_{\perp}, t\right)=\Phi_{\omega}-\Delta \Phi_{f}\left(r_{\perp}, t\right)+\Delta \Phi_{p}\left(r_{\perp}, t\right)
$$

Note also that, apart from the QE of the cathode, this modification of the work function will, furthermore, influence the energy distribution of the emitted particles. We use a linear modulation of the particles kinetic energy given by

$$
E_{k i n}\left(r_{\perp}, t\right)=E_{p}-\Phi_{\omega}^{\prime}\left(r_{\perp}, t\right),
$$


where $E_{p}$ is the final state energy of the emitted electron [42-43]. Thus, in addition to the emission charge, the space charge field affects also the initial particle energy distribution at the cathode.

\subsection{Incorporation in beam dynamics simulations}

Given a model for the QE of the emitting layer Eq. 1 - Eq. 3 and the laser intensity profile, $P_{l}\left(r_{\perp}, t\right)$, the charge produced by the cathode at time $t$ within a small time interval $\Delta t$ is given by

$$
d Q\left(r_{\perp}, t\right)=\Delta t \iint_{S} q \frac{P_{l}\left(r_{\perp}, t\right)}{h v} Q E\left(r_{\perp}, t\right) d S,
$$

where $q$ is the electron charge and $s$ denotes the emission area and $h v$ is the photon energy. This expression can be easily evaluated in beam dynamic simulations with $\Delta t$ being the time step used for particle tracking.

In order to apply Eq. 8, however, the free model parameters in Eq. 1 - Eq. 3 characterizing the cathode must be determined first. The cathode characterization procedure is exemplarily shown in Fig. 5. This procedure consists in an iterative fitting of the unknown parameters to one or more measurement data for the total emitted bunch charge. Starting with guess values for the model parameters, a tracking simulation using Eq. 8 is performed. The resulting total bunch charge is contrasted to the measured value and the emission parameters are corrected. This procedure is repeated until the simulated charge matches the measured value. The resulting model parameters characterize the given cathode and can be used in beam dynamics simulations for different injector operation conditions.

The application of this procedure for the PITZ photoinjector is shown in Fig. 6. The simulations are performed for different gun operation conditions. Three different cesiumtelluride cathodes are considered. The square point in each curve is used as reference for the cathode characterization, respectively. All other data points are computed directly by the simulations. The resulting comparisons of the total emitted bunch charge between measurement and simulation are showing the validity of the emission model for a large range of laser energies.

\subsection{Transient QE Modulation}

Given the cathode parameters, the bunch current during an emission cycle can be calculated using (Eq. 8) and the time and space dependent electromagnetic field data within the emission area. The resulting bunch current for a PITZ cathode is shown in Fig. 7 (red curve). The blue curve in the figure shows the transient modulation of the overall $\mathrm{QE}$ of the cathode resulting from the field effect (Eq. 4).

As seen in the figure, the QE of the cathode is initially higher than its integral value. This is due to the work function reduction induced by the accelerating field at the cathode, which is on the order of $0.26 \mathrm{eV}$ for an accelerating gradient of $46 \mathrm{MV} / \mathrm{m}$. Somewhere in the middle of the bunch the accelerating field is partially compensated by the build-up of space charge fields at the cathode. The space charge field effect continues to grow towards the tail of the bunch, thus, causing a further reduction of the QE. After the emission is completed, the QE of the cathode tends to increase again as the effect of space charge fields diminishes. As a result of this transient QE modulation, the temporal current profile of the bunch is no longer identical with that of the laser pulse. In this considered case, the deviation of the local current density from the flat-top laser profile is approximately $10 \%$. It should be also noted, that the response time of the semiconductor photocathode $\left(\mathrm{Cs}_{2} \mathrm{Te}\right)$ would slightly broaden or shorten the current profile depending on the RF phase. Such effect may depend also on the duration of the cathode laser 
pulse and material properties of the cathode. To take into account this effect into emission modeling, experimental characterization and determination of the cathode response time have to be performed in the near future.

A similar QE modulation effect is observed in the transverse plane. Fig. 8 shows computed QE maps at two different time instants during emission. A transverse flat-top distribution of a cylindrical cathode driving laser pulse is applied. The QE map is assumed to be homogeneous in the beginning of emission. Note that this transverse QE modulation is only due to the space charge field of the bunch at the position of the cathode. As shown, the electrons at the edge of the emission area tend to spread stronger due to the transverse space charge fields than the electrons in the central area. This lowers the particle line density in the longitudinal direction at the outer cylinder shell compared to the inner cylinder shell. Therefore, the longitudinal space charge forces at the outer cylinder shell are relatively reduced. This effect leads to the QE variation between the center and the edge of the emission area by approximately $9 \%$ (right figure) at the end of the emission for the extraction of $1 \mathrm{nC}$ bunch charge using a RMS cathode laser spot size of $0.4 \mathrm{~mm}$.

\section{Comparisons with measurements}

In Fig. 9, simulation results of the total emitted bunch charge using the LW approach (as introduced in Sec. 3) are compared with the measurement data (black curve) for the PITZ gun. The green and blue curves correspond to the usage of the Spicer (Eq. 2) and the Jensen (Eq. 3) emission models, respectively. As shown, the charge extraction behavior described by the two models agrees well over the full range of cathode laser pulse energies. For laser pulse energies lower than $45 \mathrm{~nJ}$, the simulated bunch charges are also in rather good agreements with the measured ones. This means, the incorporation of emission models with the LW approach can systematically predict the charge production in the source limited regime. Some discrepancies, however, arise as approaching to the space charge dominated regime. The measured curve slightly rises as the cathode laser pulse energy is further increased. This is neither observed in simulations nor described by the emission models. It can be resulted from the effect of surface charge limit [40-41] and/or the imperfect temporal and/or transverse distributions of the cathodeilluminating laser pulse applied in the experiments [44].

In Fig. 10, the LW simulations (red, blue and green curves) show improved agreements with the measurement data in comparisons to the conventional UMAF simulations (orange curve). The impact of nonlinear surface charge limit effects (as described in Sec. 4) on the charge extraction mechanism is also demonstrated. Two approaches are exemplarily applied for the modification of cathode work function resulting from (Eq. 5). The first approach (blue curve) uses a power index of 0.5 which is the same as given in [40]. The second one (red curve) suggests an alternative index of 0.3 . The coefficient $k$ is determined by fitting to the measurement data (black curve). As shown, using the same coefficient $k$, the latter approach renders an improved agreement with the measurement data. This nonlinear effect may be explained by the time and driving laser intensity dependencies of the cathode work function. The increase of the total work function decreases the effective QE of the cathode, lowering the portion of the total bunch charge that can be extracted from the bunch head. This results in, during first moments of the emission, a relatively lower local space charge density in the close cathode vicinity. More charges can be extracted due to a reduced longitudinal space charge field at the cathode surface. In such a nonlinear manner, the overall emission can be slightly enhanced for extracting the remaining bunch from the cathode. 


\section{Conclusion}

In this paper a three-dimensional fully electromagnetic numerical algorithm is proposed for the simulation of RF electron beam photoinjectors. Self-consistent particle emission models are incorporated within a Lienard-Wiechert (LW) approach. This made possible a dynamic electron beam generation according to the time and space dependent electromagnetic fields at the cathode and the time dependent cathode-driving laser intensity profile. The transient modulation of the cathode QE during the emission process is demonstrated according to the effect of surface barrier reduction of the emitting layer. As an application, extensive electron bunch emission simulations are performed for the PITZ injector considering various machine and beam parameters in the source limited and in the space charge limited emission regime, respectively. Fairly good agreement between measurement and simulation is achieved. Further studies are foreseen on the impacts of imperfect transverse and/or temporal distributions of the cathodedriving laser pulse on the charge extraction mechanism. Moreover, the semiconductor photocathode response time needs to be experimentally determined and the impacts of the cathode response time on dynamic process of the photoemission have to be systematically investigated in the near future. In addition, a simulation method is proposed in the Appendix for estimating the space charge emission limitation in the gun. Simulation results are presented in comparison to the measurement data for the PITZ gun.

\section{Acknowledgment}

The authors would like to sincerely thank all the colleagues from TEMF, Technische Universitaet Darmstadt and DESY in Hamburg and Zeuthen sites for all the supports and useful discussions on this topic.

\section{Appendix}

\section{A Simulation Method for Estimating Space Charge Emission Limitation}

A so-called charge iteration method is introduced to estimate the total emitted bunch charge $Q_{b}$ when the gun is operated at or close to space charge emission limitation. A simulation scheme is given in which the cathode laser pulse produces just the maximum charge that can be emitted from the cathode until the space charge limitation is reached. Using different gun phases, we start the simulation with a large initial bunch charge injected at the cathode $Q_{0}$ (e.g., $2 \mathrm{nC}$ ), and check if any macroparticles are pushed back to the cathode when the emission process is terminated. If this is the case, then we repeat the emission simulation with a lower initial $Q_{0}$. This procedure is repeated iteratively until the full bunch charge injected at the cathode $Q_{b}$ can be emitted $\left(Q_{b}=Q_{0}\right)$. In this regime, the gun is operated at or close to the space charge limit. As for simulation tool, the PIC solver of CST-PS [14] is used. A series of bunch emission simulations are performed and compared with measurements for the PITZ gun [20-21, 23].

Fig. 11 shows the total bunch charge collected at the first Integrating Current Transformer $(\mathrm{z}=0.9 \mathrm{~m})$ from the measurement (black curve) by scanning the gun operation phase for a cathode laser spot size of $0.3 \mathrm{~mm}$ (RMS). The measured bunch charge is observed to be close to the space charge limit at each gun phase. As shown, the simulation results (red curve) agree well with the measurement data. Note in addition that, for gun phases higher than 40 degrees, most particles get lost on the beam tube rather than at the cathode [45-46]. 
Fig. 12 shows a set of measurements of the total emitted bunch charge over the gun operation phase w.r.t. the phase of MMMG [20-21]. Simultaneous variations of cathode laser spot sizes, RF gun power levels and laser transmission coefficients are applied in six cases. The experimental parameters used in these measurements are shown in Table. 1. Note that, the laser transmission coefficients are set to keep the laser pulse energy same for each case. As shown, the parameter settings result in a quite similar charge extraction behavior. The emission process is space charge limited for the gun phases between -60 and 0 degrees. For the flat region between 0 and 50 degrees, the total bunch charge does not strongly depend on the gun phase. This indicates the source limited emission. All the measurements in Fig. 12 are compared with the CST-PS simulations in Fig. 13. As shown, the black curves represent the measurement data, while the red curves show the space charge limits calculated by the simulations. In addition, the pink curves indicate the charge extraction behavior when the gun is operated with the laser pulse energies below the space charge limit for gun phases between 0 and 50 degrees.

\begin{tabular}{cccc} 
Table 1: Experimental parameters for the six measurements in Fig. 12. \\
\cline { 2 - 4 } Case & $\begin{array}{c}\text { RMS laser } \\
\text { spot size } \\
(\mathrm{mm})\end{array}$ & $\begin{array}{c}\text { Gun } R F \\
\text { power } \\
(\mathrm{MW})\end{array}$ & $\begin{array}{c}\text { Laser } \\
\text { transmission } \\
\text { coefficient (\%) }\end{array}$ \\
\hline 1 & 0.302 & 6.5 & 57.0 \\
2 & 0.312 & 6.0 & 52.6 \\
3 & 0.327 & 5.4 & 48.2 \\
4 & 0.341 & 5.0 & 43.8 \\
5 & 0.361 & 4.6 & 39.5 \\
6 & 0.381 & 4.0 & 35.1 \\
\hline
\end{tabular}

\section{References}

[1] F. Stephan, M. Krasilnikov, High Brightness Photo Injectors for Brilliant Light Sources, in: E. Jaeschke, S. Khan, J. R. Schneider, J. B. Hastings, Synchrotron Light Sources and FreeElectron Lasers, 1st ed., Springer International Publishing Switzerland, 2016, ISBN9783319143941.

[2] M. Krasilnikov, F. Stephan, G. Asova, H. Grabosch, M. Gross, L. Hakobyan, I. Isaev, Y. Ivanisenko, et al., Experimentally minimized beam emittance from an L-band photoinjector, Phys. Rev. ST Accel. Beams, 15 (2012), 100701.

[3] F. Stephan, C. Boulware, M. Krasilnikov, J. Baehr, G. Asova, A. Donat, U. Gensch, H. Grabosch, et al., Detailed characterization of electron sources yielding first demonstration of European X-ray Free-Electron Laser beam quality, Phys. Rev. ST Accel. Beams, 13 (2010), 020704. 
[4] W. Ackermann, G. Asova, V. Ayvazyan, A. Azima, N. Baboi, J. Baehr, V. Balandin, B. Beutner, et al., Operation of a free-electron laser from the extreme ultraviolet to the water window, Nature Photon 1 (2007), 336-342.

[5] D. Dowell, E. Jongewaard, J. Lewandowski, C. Limborg-Deprey, Z. Li, J. Schmerge, A. Vlieks, J. Wang, L. Xiao, The development of the linac coherent light source RF gun, arXiv 1503.05877 (2015).

[6] D. Dowell, I. Bazarov, B. Dunham, K. Harkay, C. Hernandez.Garcia, R. Legg, H. Padmore, T. Rao, J. Smedley, W. Wan, Cathode R\&D for future light sources, Nucl. Instrum. Methods Phys. Res. A 622 (2010), 685-697.

[7] R. Akre, D. Dowell, P. Emma, J. Frisch, S. Gilevich, G. Hays, P. Hering, R. Iverson, et al., Commissioning the linac coherent light source injector, Phys. Rev. ST Accel. Beams 11 (2008), 030703.

[8] T. Schietinger, M. Pedrozzi, M. Aiba, V. Arsov, S. Bettoni, B. Beutner, M. Calvi, P. Craievich, et al., Commissioning experience and beam physics measurements at the SwissFEL Injector Test Facility, Phys. Rev. Accel. Beams 19 (2016), 100702.

[9] W. Brefeld, B. Faatz, Y. Nikitina, J. Pflueger, P. Pierini, J. Rossbach, E. Saldin, E. Schneidmiller, M. Yurkov, Parameter study on phase I of the VUV-FEL at the TESLA test facility, Nucl. Instrum. Methods Phys. Res. A 393 (1997), 119-124.

[10] P. Schmueser, M. Dohlus, J. Rossbach, Ultraviolet and Soft X-Ray Free-Electron Lasers: Introduction to Physical Principles, Experimental Results, Technological Challenges, SpringerVerlag, Berlin, 2008, ISBN9783540795728.

[11] C. Limborg, Y. Batygin, J.-P. Carneiro, K. Floettmann, L. Giannessi, M. Quattromini, M. Boscolo, M. Ferrario, V. Fusco, C. Ronsivalle, Code comparison for simulations of photoinjectors, in: Proc. Particle Accelerator Conference, Portland, Oregon, USA, 2003, pp. 35483550 .

[12] E. Colby, V. Ivanov, Z. Li, C. Limborg, Simulation issues for RF photoinjectors, in: Proc. 7th International Computational Accelerator Physics Conference, Michigan, USA, 2002.

[13] E. Gjonaj, Numerical modeling of rf electron sources for fel-accelerators, in: Proc. 11th International Computational Accelerator Physics Conference, Rostock-Warnemünde, Germany, 2012.

[14] C. S. T. AG, CST-PS: Computer Simulation Technology Particle Studio, http://www.cst.de.

[15] K. Floettmann, ASTRA: A Space Charge Tracking Algorithm, http://www.desy.de/mpyflo/.

[16] L. M. Young, PARMELA: Phase and Radial Motion in Electron Linear Accelerators, http://laacg.lanl.gov/laacg/services/. 
[17] J. Qiang, IMPACT-T: A 3D Parallel Particle Tracking Code in Time Domain, http://portal.nersc.gov/project/m669/IMPACT-T/.

[18] S. B. van der Gee, M. J. de Loos, GPT: the General Particle Tracer (GPT) package, http://www.pulsar.nl/.

[19] L. Giannessi, M. Quattromini, TREDI, http://www.tredi.enea.it/.

[20] M. Krasilnikov, Tech. Rep., DESY PUBDB-2015-05913, 2015, https://bibpubdb1.desy.de/record/292200.

[21] M. Krasilnikov, Tech. Rep., DESY PUBDB-2014-00349, 2013, https://bibpubdb1.desy.de/record/165339.

[22] E. Gjonaj, Tech. Rep., DESY PUBDB, 2011, http://www.desy.de/fel-beam/.

[23] M. Krasilnikov, Problems observed at PITZ: measurements vs. simulations, in: Proc. ICFA Workshop on Future Light Sources, Thomas Jefferson National Accelerator Facility, Newport News, VA, USA, 2012.

[24] J. Li, G. Asova, M. Gross, L. Hakobyan, I. Isaev, Y. Ivanisenko, M. Khojoyan, G. Klemz, G. Kourkafas, M. Krasilnikov, et al., in: Proc. 11th International Computational Accelerator Physics Conference, Rostock-Warnemünde, Germany, 2012, pp. 242-244.

[25] R. Ryne, C. Mitchell, J. Qiang, et al, Using a Lienard-Wiechert solver to study coherent synchrotron radiation effects, in: Proc 35th International Free-Electron Laser Conference, New York, NY, USA, 2013, pp.17-23.

[26] F. Ciocci, L. Giannessi, A. Marranca, L. Mezi, M. Quattromini, Self-consistent threedimensional RF-gun dynamics integration based on the Lienard-Wiechert retarded potentials, Nucl. Instrum. Methods Phys. Res. A 393 (1997), 434-438.

[27] W. E. Spicer, Photoemissive, photoconductive, and optical absorption studies of alkaliantimony compounds, Phys. Rev. 112 (1958), 114-122.

[28] W. E. Spicer, Photoemission and related properties of the alkali-antimonides, Journal of Applied Physics 31 (1960), 2077-2084.

[29] W. E. Spicer, F. Wooten, Photoemission and photomultipliers, in: Proc. the IEEE, vol. 51, 1963, pp. 1119-1126.

[30] W. E. Spicer, A. Herrera-Gomez, Modern theory and applications of photocathodes, in: Proc. SPIE, vol. 2022, San Diego, CA, USA, 1993, pp. 18-35. 
[31] L. A. DuBridge, Theory of the energy distribution of photoelectrons, Phys. Rev. 43 (1933), 727-741.

[32] W. Wendelen, B. Y. Mueller, D. Autrique, B. Rethfeld, A. Bogaerts, Space charge corrected electron emission from an aluminum surface under non-equilibrium conditions, Journal of Applied Physics 111 (2012), 113110.

[33] M. Cardona, L. Ley, Photoemission in Solids 1, Springer International Publishing Switzerland, 1978, ISBN 978-3-540-35895-4.

[34] K. L. Jensen, Electron Emission Physics, in: Peter W. Hawkes, Advances in Imaging and Electron Physics Vol. 149, Springer International Publishing Switzerland, 2007, ISBN 978-0-12374207-0.

[35] K. L. Jensen, B. L. Jensen, E. J. Montgomery, D. W. Feldman, P. G. O'Shea, N. Moody, Theory of photoemission from cesium antimonide using an alpha-semiconductor model, Journal of Applied Physics 104 (2008), 044907.

[36] K. L. Jensen, N. A. Moody, D. Feldman, E. J. Montgomery, P. G. O'Shea, Photoemission from metals and cesiated surfaces, J. Appl. Phys. 102 (2007), 074902.

[37] K. L. Jensen, J. J. Petillo, D. N. Panagos, S. Ovtchinnikov, N. A. Moody, Delayed photoemission model for beam optics codes, J. Vac. Sci. Technol. B 35 (2017), 02 C102.

[38] D. Dowell, J. Schmerge, Quantum efficiency and thermal emittance of metal photocathodes, Phys. Rev. ST Accel. Beams 12 (2009), 074201.

[39] J. Schmerge, J. Clendenin, D. Dowell, S. Gierman, RF gun photo-emission model for metal cathodes including time dependent emission, Int. J. Mod. Phys. A 22 (2007), 4069-4082.

[40] M. Zolotorev, Tech. Rep., SLAC-PUB-5896, 1992, http://www.slac.stanford.edu/cgiwrap/getdoc/slac-pub-5896.pdf.

[41] M. Woods, J. Clendenin, J. Frisch, A. Kulikov, P. Saez, D. Schultz, J. Turner, K. Witte, M. Zolotorev, Tech.Rep., SLAC-PUB-5894, 1992, https://www.slac.stanford.edu/pubs/slacpubs/5750/slac-pub-5894.pdf.

[42] R. A. Powell, W. E. Spicer, G. B. Fisher, P. Gregory, Photoemission studies of cesium telluride, Phys. Rev. B 8 (1973), 3987-3995.

[43] K. Floettmann, Tech. Rep., TESLA-FEL 97-01, 1997, https://flash.desy.de/sites/site_vuvfel/content/e403/e1642/e839/e829/infoboxContent830/teslafel1997-01.pdf. 
518 [44] C. H. Garcia, M. Krasilnikov, G. Asova, M. Bakr, P. Boonpornprasert, J. Good, M. Gross, 519 H. Huck, I. Isaev, D. Kalantaryan, et al., Charge production studies from Cs2Te photocathodes in a normal conducting RF gun, Nucl. Instrum. Methods Phys. Res. A 871 (2017), 97-104. simulations of the PITZ photoinjector, in: Proc. 5th International Particle Accelerator Conference, Dresden, Germany, 2014, pp. 391-393.

[46] Y. Chen, H. D. Gersem, E. Gjonaj, A. Tsakanian, T. Weiland, C. Hernandez-Garcia, M. XFEL photoinjector at DESY-PITZ, in: Proc. 6th International Particle Accelerator Conference, Virginia, USA, 2015, pp. 162-164. 


\section{Color should be used for all figures except for Fig. 5 .}

Fig. 1. Sketch of the PITZ RF gun. Left: Exterior view. Right: Cut-plane view depicting the 1.6-cell cavity and the RF waveguide system.

Fig. 2. Measured and simulated bunch charges for different gun phases of the PITZ injector. A flat-top laser pulse of 20 ps length and a RMS spot size of $0.3 \mathrm{~mm}$ are applied. Two laser pulse intensities are considered, denoted by LT $=100 \%$ and $\mathrm{LT}=62 \%$, respectively.

Fig. 3. Bunch charge measured at the ICT1 $(z=0.95 \mathrm{~m})$ as a function of the representative cathode laser pulse intensity for $\mathrm{XYrms}=0.3 \mathrm{~mm}$ and the MMMG gun phase. The red and blue curves correspond to the measured and simulated charge, respectively. Note, furthermore, that in the simulation, the input bunch charge provided at the cathode, $Q_{0}$, was assumed to grow proportionally with laser pulse intensity, and thus chosen to represent the cathode laser intensity as shown on the horizontal axis of the figure. For a source limited emission, the emitted bunch charge is identical with the input charge at the cathode. Thus, the linear run of the curve at lower laser intensities does not represent a simulation result but, rather, the assumption used in it.

Fig. 4. Comparisons of the total emitted bunch charge between EM-PIC (CST-PS) simulation, LW simulation and UMAF simulation for a RMS cathode laser spot size XYrms $=0.3 \mathrm{~mm}$. The maximum RF field gradient is about $60 \mathrm{MV} / \mathrm{m}$. A flat-top temporal profile of the cathode laser pulse is applied (about 21.5 ps FWHM). Convergence of EM-PIC simulations are checked using improved mesh resolutions, $\mathrm{dz}=20,10$ and $5 \mu \mathrm{m}$. $\mathrm{dz}$ represents the minimum mesh step in the longitudinal direction. This results in overlapping dotted blue, green and red curves. $Q_{0}$ is the initial bunch charge injected at the cathode.

Fig. 5. Schematic of the photocathode characterization procedure. $t_{\text {end }}$ is the total emission time, $Q_{m e s}$ is the measured total bunch charge used as reference.

Fig. 6. Emitted bunch charge versus laser pulse energy for three different cathodes.

Fig. 7. Transient bunch current and modulation of the cathode QE due to surface barrier reduction of the emitting layer for a temporally flat-top laser profile (approx. 18 ps FWHM) at the cathode. The maximum accelerating electric field gradient is about $46 \mathrm{MV} / \mathrm{m}$

Fig. 8. Transient modulation of the cathode $\mathrm{QE}$ on the transverse plane of emission at different time instants. The total emission time is about 30 ps. Left figure: $t=16$ ps; Right figure: $t=30$ ps. The time instants are in respect to the starting time-clock of the emission process, 0 ps.

Fig. 9. Comparisons of total emitted bunch charges between LW simulation and measurement. The RF gun power is about 1.5 MW and the cathode-illuminating laser pulse has a temporal Gaussian profile of about $1.5 \mathrm{ps}$ (RMS). The black curve represents the measurement data for a cathode with an integral QE of $8.5 \%$.

Fig. 10. LW simulations with nonlinear surface charge limit effects on and comparisons with the measurement data.

Fig. 11. The total emitted bunch charge as a function of the gun phase w.r.t. the phase of MMMG for a RMS laser spot size of 0.3 $\mathrm{mm}$. The maximum RF field gradient is $60 \mathrm{MV} / \mathrm{m}$. A temporally $21.5 \mathrm{ps}$ flat-top prole of the laser pulse is applied.

Fig. 12. Simultaneous variation of multiple gun operation parameters for the measurements of the total bunch charge over the gun phase. The parameter XYrms represents the RMS size of the cathode laser spot, while $P_{r f}$ denotes the RF power in the gun. The laser transmission coefficient $(L T)$ characterizes the relative laser intensity at the cathode.

Fig. 13. Comparisons of the emitted bunch charge between the measurements and the CST-PS simulations under variable experimental conditions of the gun. XYrms represents the RMS laser spot width. $P_{r f}$ represents the RF gun power. $L T$ indicates the laser transmission coefficient. Cases 1 to 6 correspond to the measured curves in Fig. 12, respectively. 


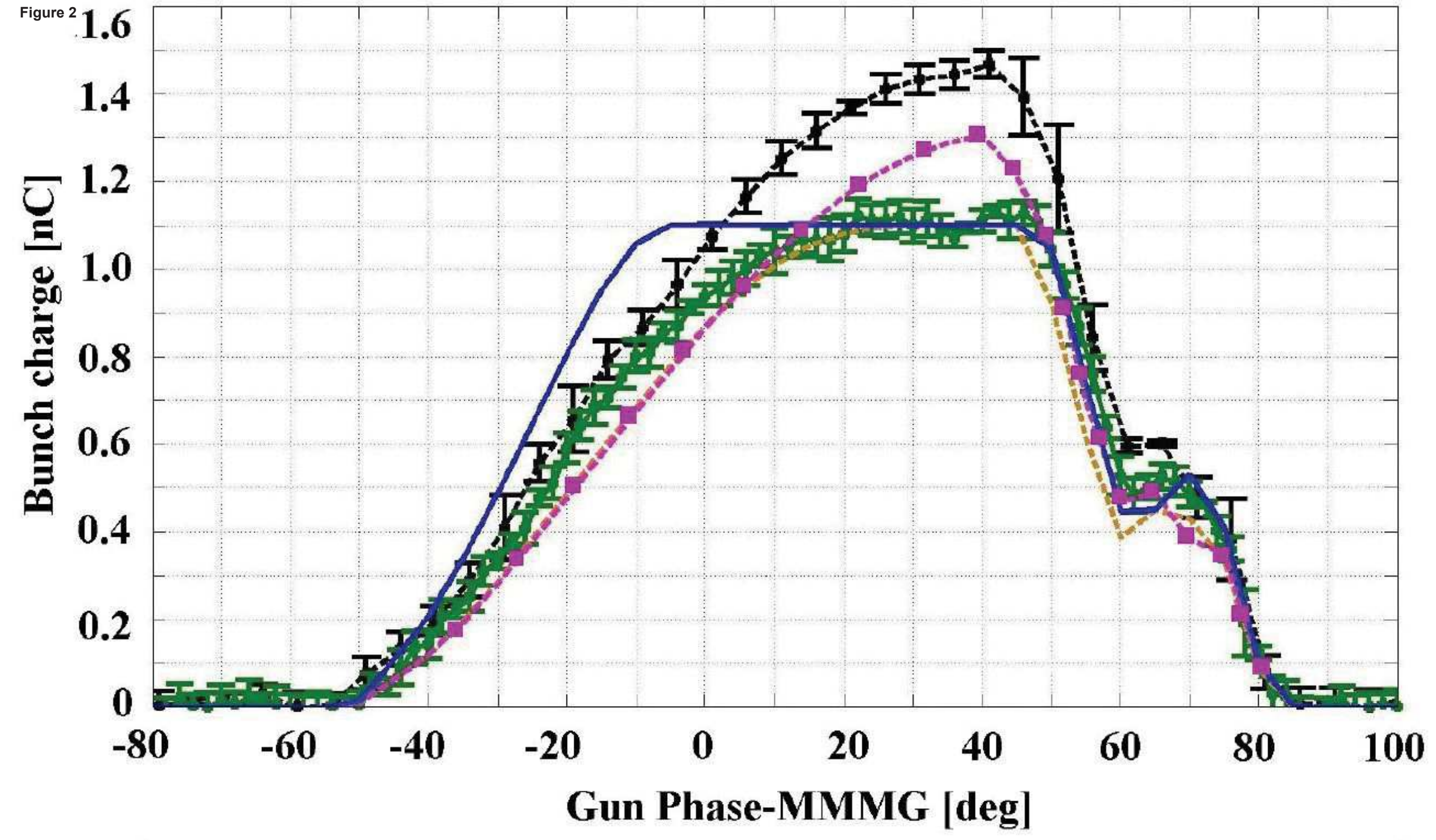

$\rightarrow-\infty$ Measured charge $(X Y \mathrm{rms}=0.3 \mathrm{~mm}, \mathrm{LT}=100 \%)$

$\Rightarrow$ Measured charge $(X Y r m s=0.3 \mathrm{~mm}, \mathrm{LT}=62 \%)$

---- Simulated charge $\left(X Y \mathrm{rms}=0.3 \mathrm{~mm}, \mathrm{Q}_{0}=1.1 \mathrm{nC}\right.$ for $\left.\mathrm{LT}=62 \%\right)$

$=-$ Simulated charge $\left(X Y \mathrm{rms}=0.3 \mathrm{~mm}, \mathrm{O}_{0}=1.774 \mathrm{nC}\right.$ for $\mathrm{LT}=100 \%$ )

- Simulated charge $(X Y r m s=0.4 \mathrm{~mm}, \mathrm{Q} 0=1.1 \mathrm{nC}$ for $\mathrm{LT}=62 \%)$ 


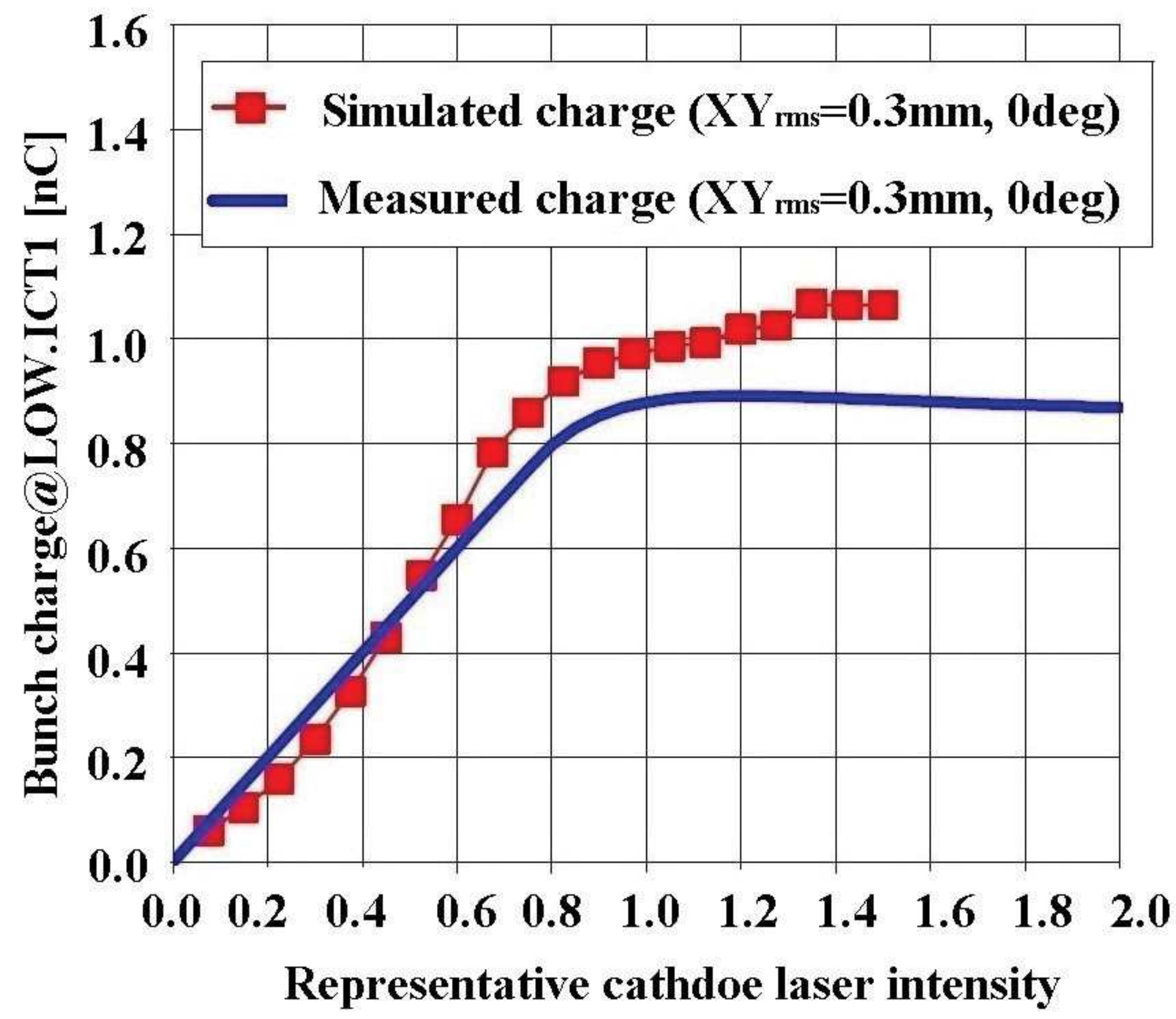




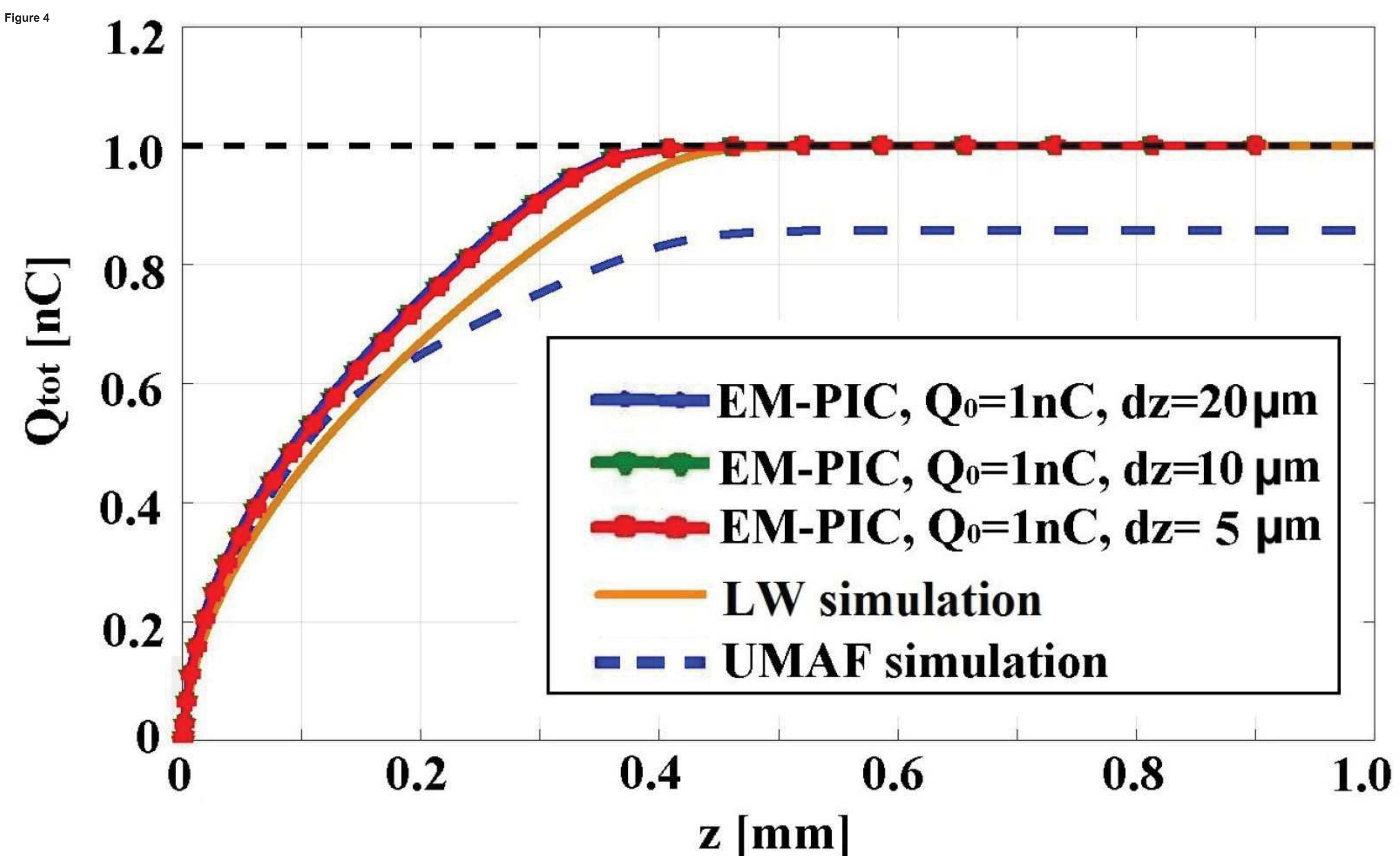




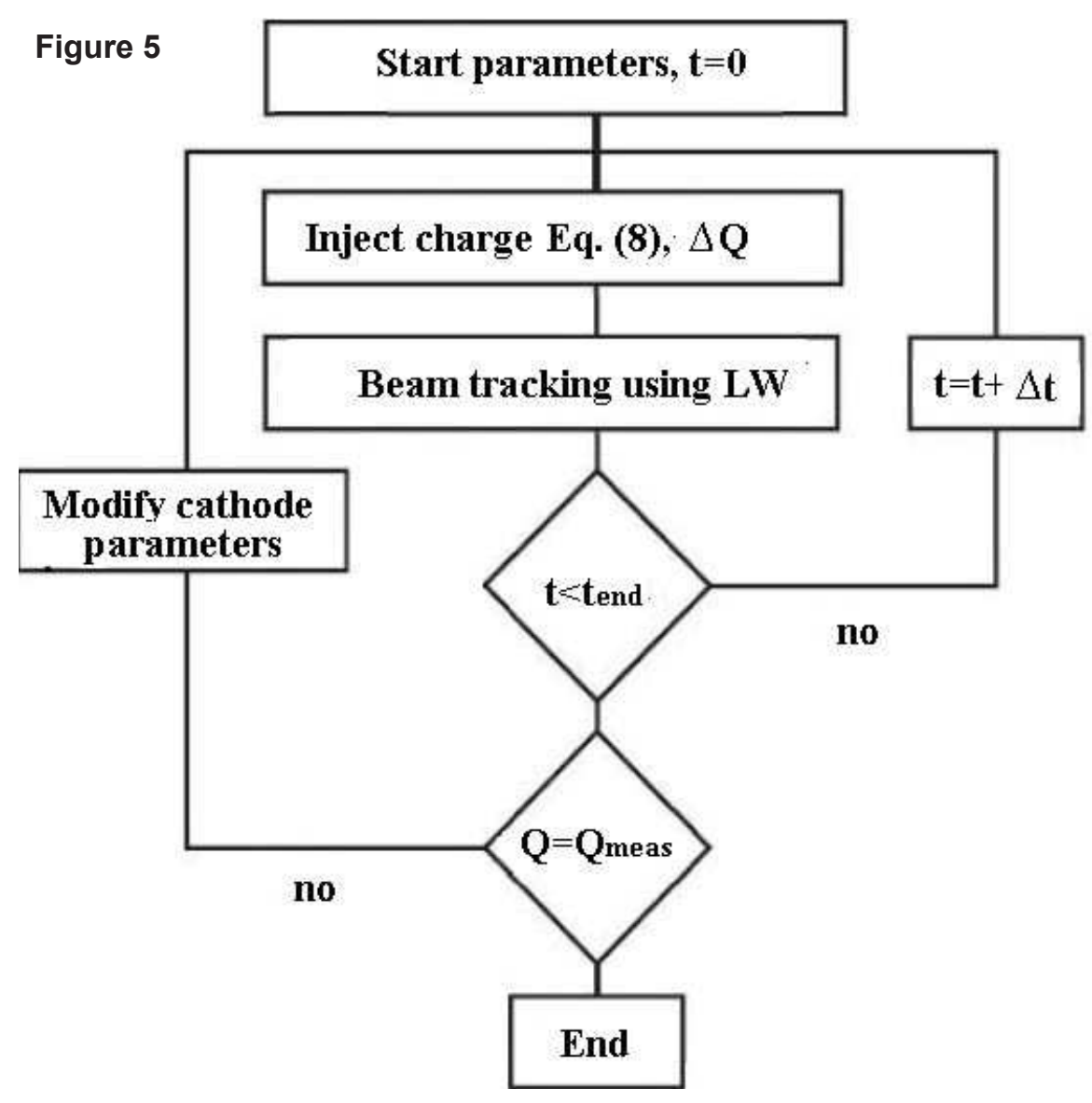




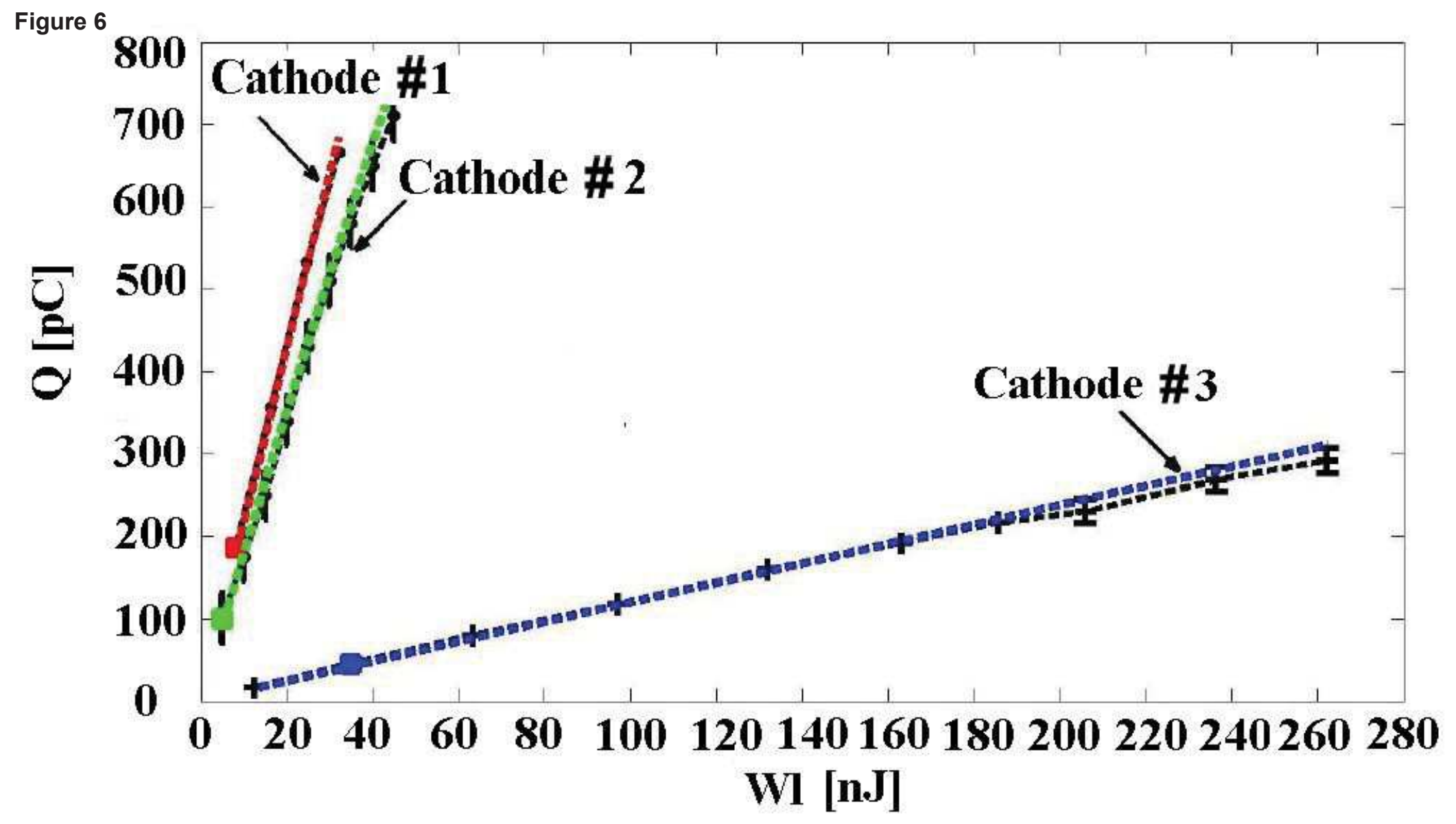

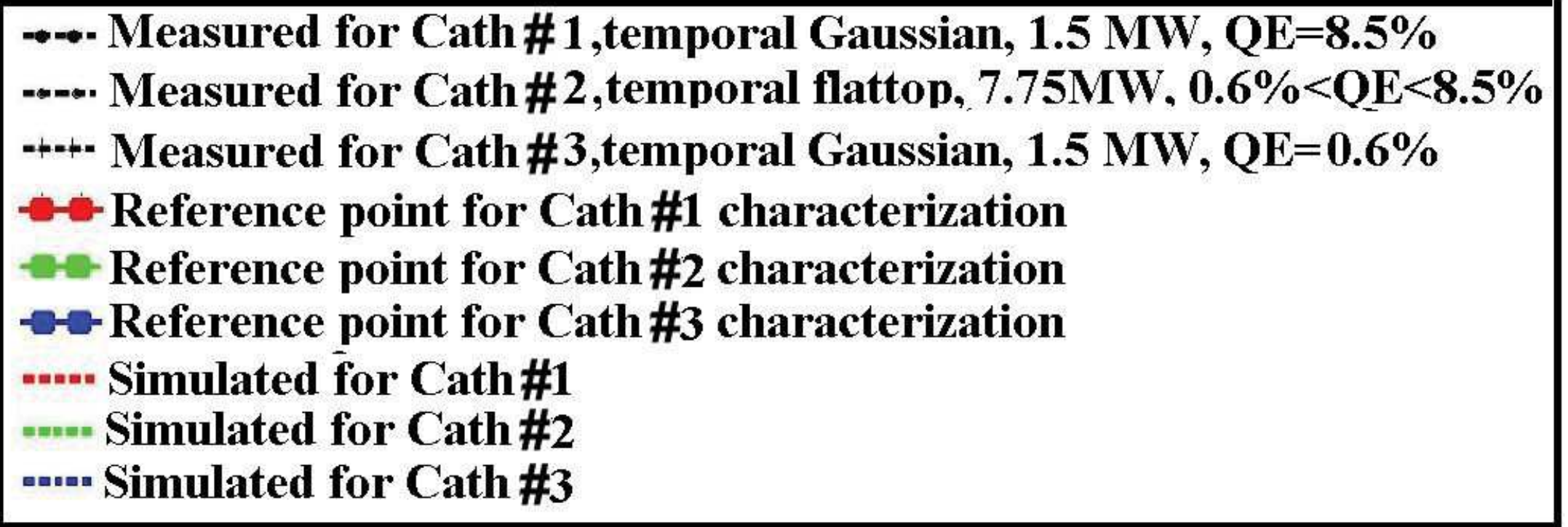




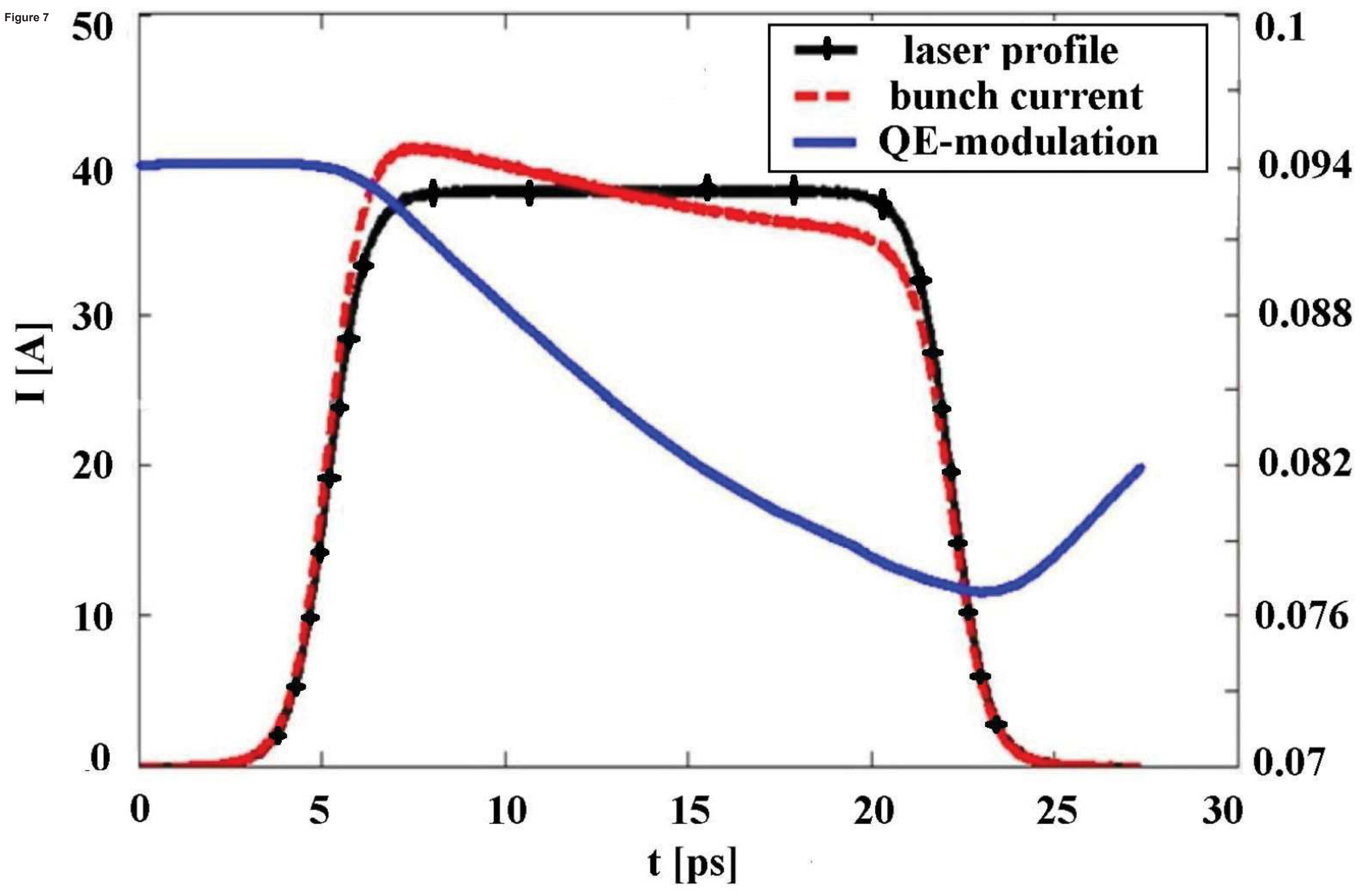



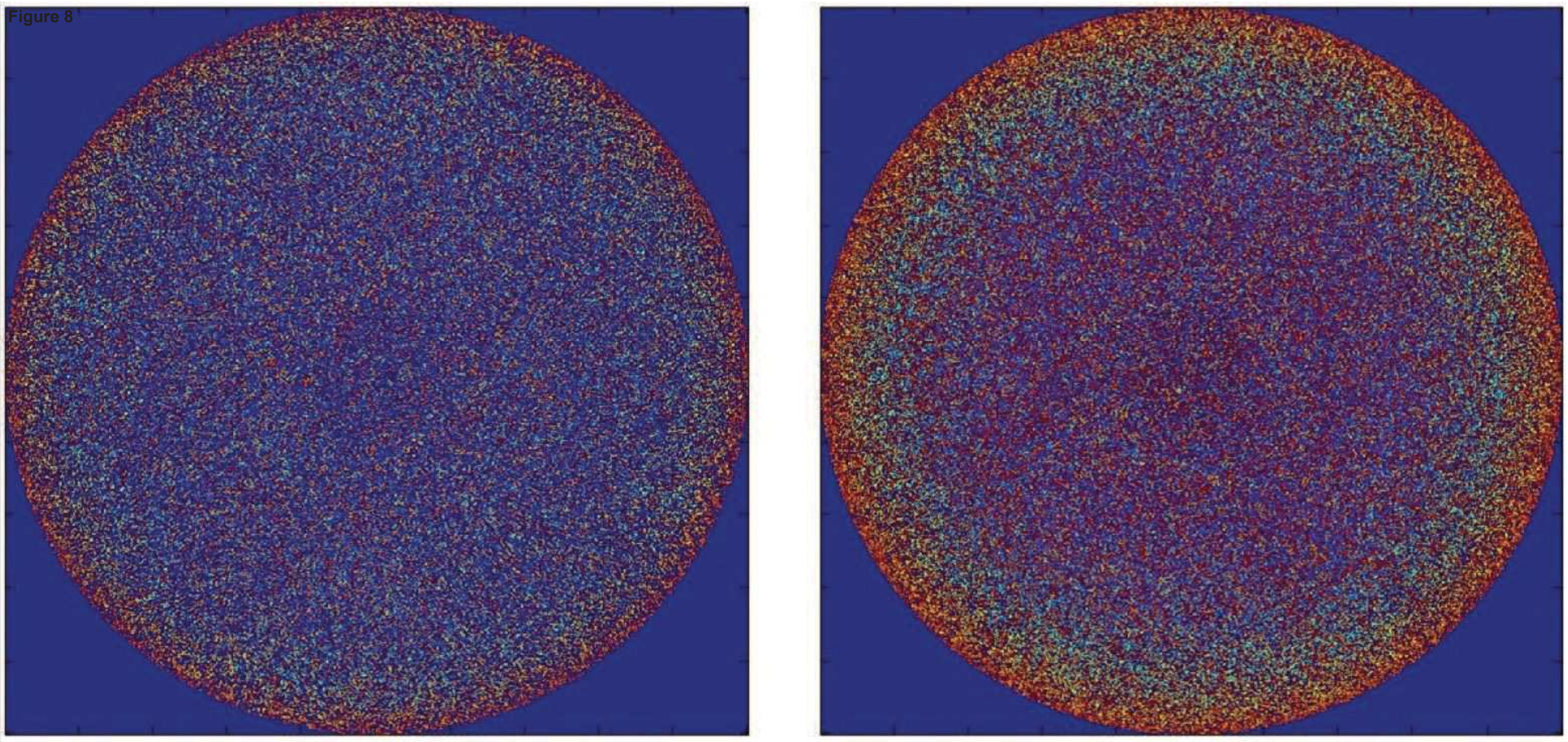

0.08

0.082

0.084

0.078

0.08

0.082

0.084 


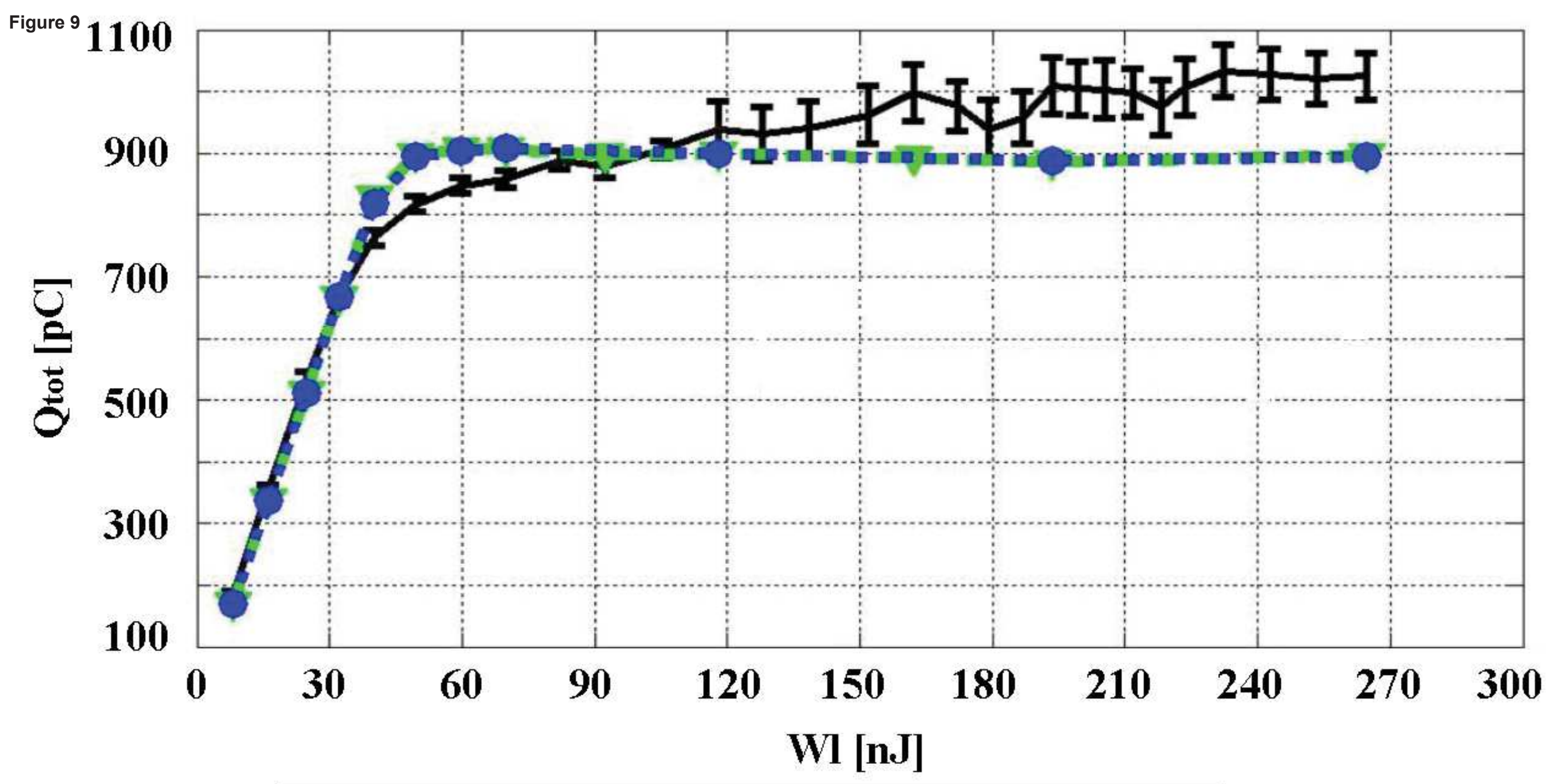

- Measurement data

- $\mathrm{LW}$ simulation with the Spicer model

-O-OW simulation with the Jensen model 


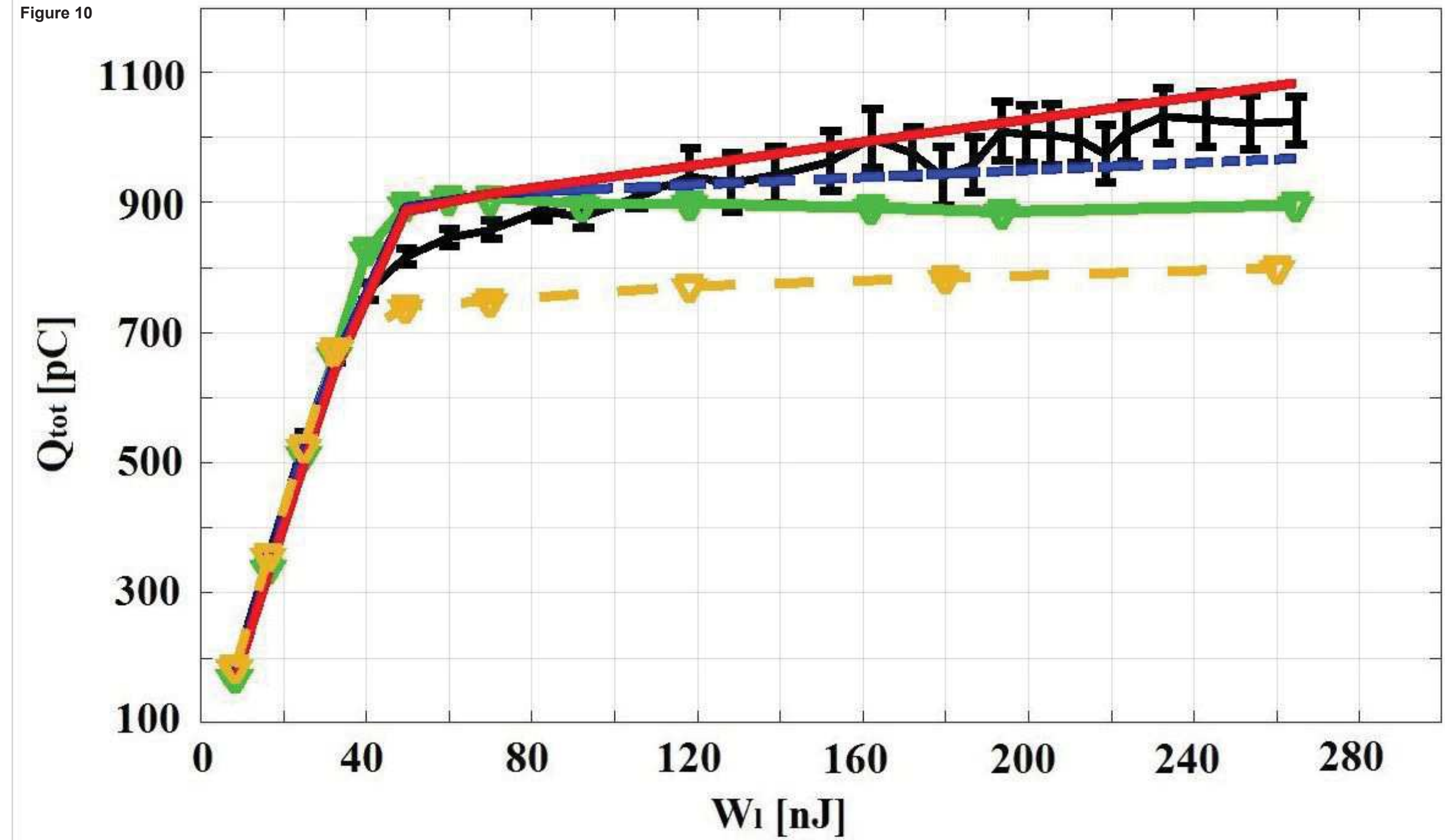

$\longrightarrow$ Measurement, $\mathrm{QE}=8.5 \%$

$\sim \quad L W$ simulation without $\Delta \Phi_{p}$

LW simulation with $\Delta \Phi_{\mathrm{p}} \sim 1.2 *[\mathrm{I}(\mathrm{t}, \mathrm{r})]^{0.5}$

LW simulation with $\Delta \Phi_{\mathrm{p}} \sim 1.2 *[\mathrm{I}(\mathrm{t}, \mathrm{r})]^{0.3}$

$\checkmark$ Conventional UMAF simulations 


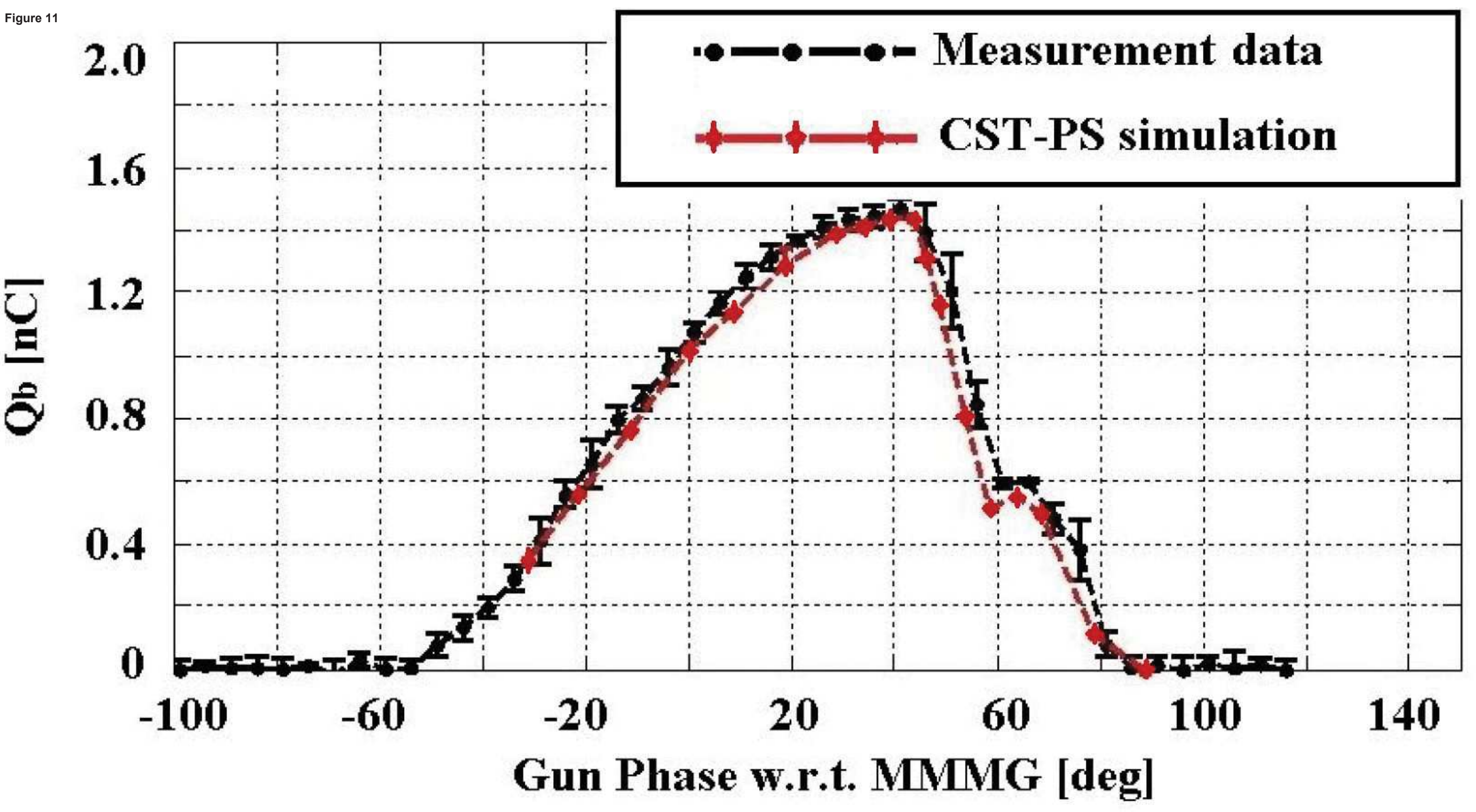




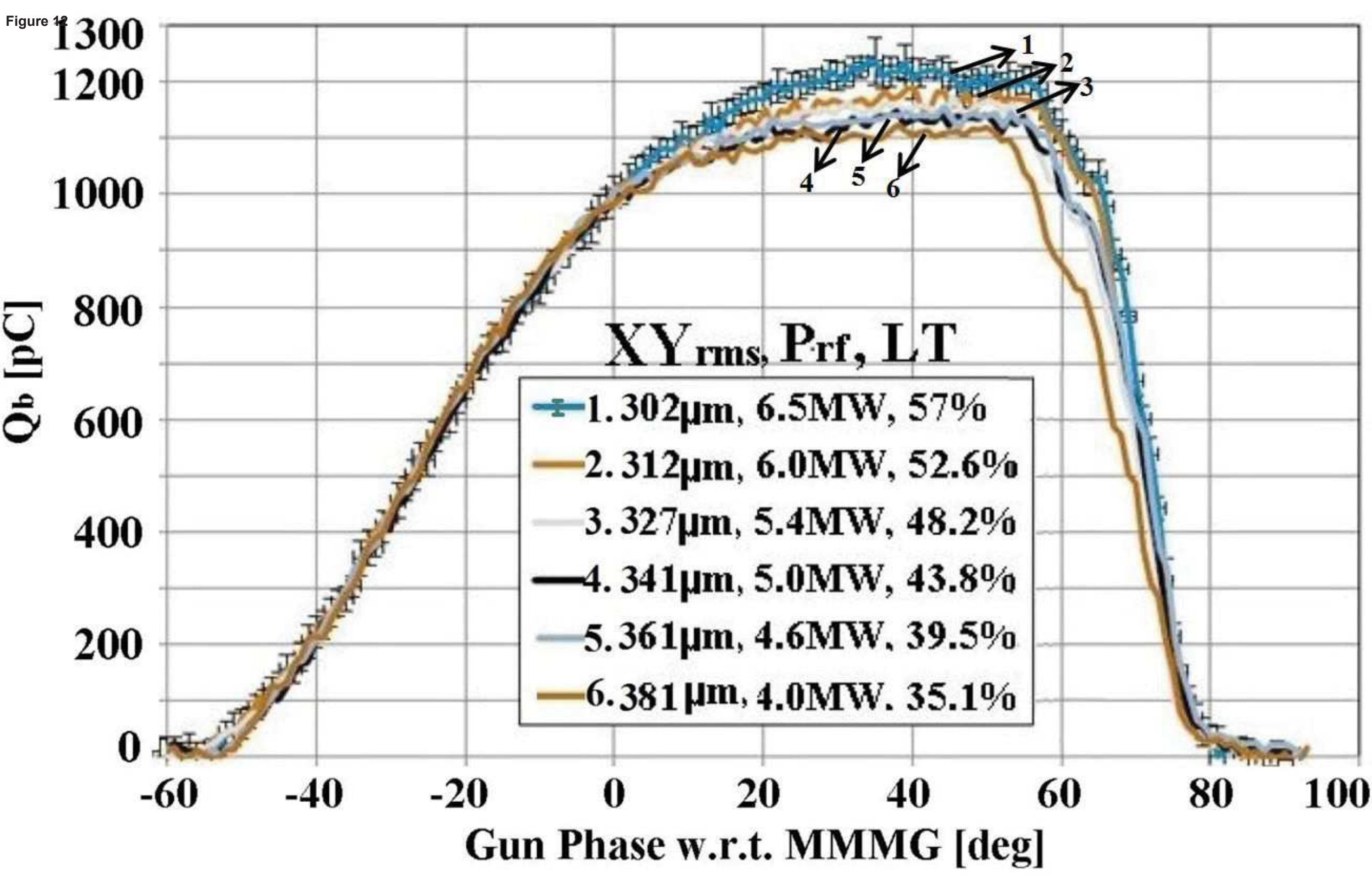


